\title{
Comparing convection in the Sun with Procyon A
}

\author{
Frank Robinson ${ }^{1}$ and Pierre Demarque ${ }^{1}$ \\ ${ }^{1}$ Department of Astronomy, Yale University, New Haven, CT 12345, USA \\ email: frank.robinson@yale.edu
}

\begin{abstract}
As well as reproducing observable features like solar granulation, 3D simulations can provide useful information for local or non-local stellar modeling. Examples include testing out the mixing length theory in the surface layers, providing the turbulent correction for stellar models of $p$-mode frequencies, testing eddy viscocity prescriptions used in tidal dissipation models and comparing different closures of higher order moments. Having validated the $3 \mathrm{D}$ code with the sun we applied it to other stars. For Procyon A the turbulence is about twice as strong as it is in the Sun (estimated by the peak root mean square vertical velocity which is about $6.5 \mathrm{~km} / \mathrm{s}$ ) and the granules are nearly an order of magnitude larger, with plumes regularly extending down 2-3 pressure scale heights below the surface of Procyon A. An interesting feature of the Procyon simulation is that the horizontal layers in the vicinity of the granules appear to radially pulsate in a quasi-periodic manner. This makes the superadiabatic layer move in and out over a distance of about half a pressure scale height in a time of about 20-30 minutes. This motion appears to be tied to the granulation but there may be other phenomena involved such as some kind of local kappa-mechanism confined to the granulation surface layers. We find similar behaviour in a simulation of an 11 Gyr subgiant sun. As the velocities are more like the sun (about $3 \mathrm{~km} / \mathrm{s}$ ), the time period of the pulsation in the subgiant is 2-3 times longer than in Procyon A.
\end{abstract}

Keywords. Sun: atmosphere, hydrodynamics

\section{Introduction}

Convection is ubiquitous in nature and yet a general theory still does not exist. 3D simulations provide a means of investigating certain convective phenomena for which an analytical solution has not yet been found. In each case the governing equations are the Navier-Stokes equations which are solved on a computer with certain terms being included/excluded depending on the situation. In special cases where the flow is smooth (laminar) simulations are able to resolve all the dynamical scales (from the viscous dissipation scale of a few $\mathrm{mm}$ up to the biggest eddies of say a few $\mathrm{cm}$ in laboratory experiments). In this case computed convection characteristics such as bifurcation points, frequencies and velocities closely match laboratory experiments (Robinson \& Chan 2004). While such simulations are non-linear they only encompass a small range of scales (of order 10) and have Reynolds numbers of typically less than 100. As the degree of nonlinearity is increased $\mathbf{u} \cdot \nabla \mathbf{u}$ becomes large compared to $\nu \nabla^{2} \mathbf{u}$ and the range of scales and unpredictability increases until the flow becomes turbulent.

The earth's atmosphere which is stably stratified exhibits intermittent turbulence ("the fasten seatbelt sign goes on only sometimes during a flight") often associated with deep moist convection and the formation of clouds. Large eddy simulations can be used to resolve a small part of the inertial range so that most of the turbulent energy is in the resolved scales and smaller scales are parameterised by a subgrid model. Such simulations can be compared to cloud-top heights or vertical velocities from satellite, balloon or 
aircraft data. Though most simulations tend to overestimate updraft speeds in the upper troposphere (Robinson \& Sherwood 2006).

For simulating convection zones in stars the main source of accurate data is the sun. Simulations of the surface layers of the sun have successfully reproduced solar sized granules (Stein \& Nordlund 1998), and computed $p$-mode frequencies within a few $\mu \mathrm{Hz}$ of observed values ( $\mathrm{Li}$ et al. 2002). It is particularly encouraging that realistic solar simulations employing different input physics and having different upper/lower boundary conditions seem to get similar (within 10\%) results away from the boundaries (Kupka 2005). This is true provided the numerical resolution is sufficient - which is becoming possible for a greater range of applications because of significant increases in computing power.

\section{Modeling realistic stellar surface convection}

\subsection{Geometry}

Due to the huge range of scales in the solar convection zone the computational domain was restricted to a tiny box located at the top of the convection zone. The box had a width equal to that of a few granules and a depth of at least 5 pressure scale heights (PSH), with the top of the box located about $1 \mathrm{PSH}$ above the photosphere. The size of the domain is important as boundary effects can be reduced (though never eliminated) by increasing the size of the box until quantities within 1 PSH of the upper and lower boundaries remain unchanged if further increases in size of the box are made. The vertical walls were periodic and the horizontal walls free slip and impenetrable (closed box). The resolution should be enough so that at least part of the inertial range is included in the simulation and most of the turbulent kinetic energy is in the resolved scales $(10-1000 \mathrm{~km}$ for the sun).

\subsection{Realistic physics}

Our numerical approach can be summarized as follows (see Robinson et al. (2003) for more details):

1. A standard Solar model, 1D Yale Stellar Evolution model (Guenther \& Demarque 1997) was used to compute initial stratification.

2.Alexander low temperature opacities, OPAL opacities and Equation of State. Hydrogen and Helium ionizations zones were included.

3. We performed a large eddy simulation of the full Navier-Stokes equations in the small box with constant acceleration due to gravity and no rotation (Kim \& Chan 1998). Similar opacities and equation of state to those in the 1D stellar model were used in the simulation.

4.Radiative energy transport was modeled by diffusion approximation in deep layers and 3D Eddington approximation for a grey atmosphere in shallow regions (Unno \& Spiegel 1966).

\section{Applications of 3D solar simulations}

What information can be extracted from the $3 \mathrm{D}$ simulations that is of use to the theoreticians or observers in the stellar community?

(a) Testing the mixing length theory in the surface layers.

In deep efficient mixed convection the mixing length theory (MLT) has been found to work very well (Chan \& Sofia 1989). However, near the surface of the sun partial hydrogen ionization and low gas density reduce the efficiency of convective heat transport compared 

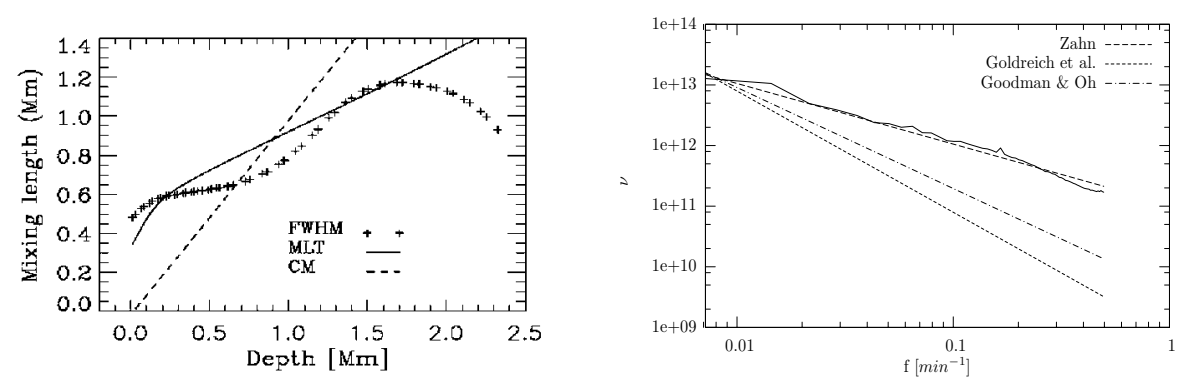

Figure 1. Left panel: Prescriptions for mixing length. Right panel: Comparison of different eddy viscocity models with simulation(solid line).

to that by radiation. This region is called the superadiabatic layer (SAL). In the SAL, the validity of mixing length theory is less certain. To estimate the mixing length in the simulations we use the full width at half maximum (FWHM) of the auto-correlation of the vertical velocity. This metric tells us approximately how far a convective element moves radially before mixing with the surrounding fluid. The left panel of Fig. 1 shows the FWHM for the solar simulation (denoted by crosses) versus distance from the surface of unit optical depth along with two other prescriptions for the mixing length, namely the standard $\alpha H_{\mathrm{p}}$ (denoted MLT) where $\alpha$ is the solar calibrated value and $\mathrm{H}_{\mathrm{p}}$ is the pressure scale height and the distance inwards from the solar surface denoted CM Canuto \& Mazzitelli (1991). In the SAL the simulation mixing length doesn't match either MLT or CM. The bending of the curve at around $1.6 \mathrm{Mm}$ is a result of the lower boundary and it seems reasonable to say that if the boundary were not there the simulation would asymptote to the mixing length value. If the same procedure is applied to non-solar simulations then the differences between simulation and MLT seem to increase and a different value of $\alpha$ is required to match the simulation mixing length in the deep adiabatic part of the simulation.

(b) Incorporating turbulence into stellar models.

One of the success of 3D simulations (Stein \& Nordlund 2000) has been to partly explain the discrepancy between the predicted value of $p$-mode frequencies in the surface layers and the values observed in helioseismology. Li et al. (2002) incorporated the mean turbulent pressure and turbulent kinetic energy into a stellar model and found that the recomputed frequencies were much closer to observations. In Straka et al. (2006) they show that applying a similar procedure to a model of $\eta$-Bootis also brings the model frequencies much closer observations (also see poster S239-80 for the application to $\alpha$-cen).

(c) Tidal dissipation in stars (Penev et al. 2006).

If tidal dissipation is modeled as a viscous dissipation process then one question is which model to use for the eddy viscoscity. The model proposed by Zahn (1966) assumes a linear scaling $\nu=\nu_{\max } \max [\mathrm{T} / 2 \tau, 1]$, while Goldreich \& Keeley (1977) and Goodman \& Oh (1997) propose a quadratic scaling $\nu=\nu_{\max } \max \left[(\mathrm{T} / 2 \pi \tau)^{2}, 1\right]$, where $\mathrm{T}$ and $\tau$ are the perturbation period and eddy turnover time. Using an FFT of the 3D solar surface velocity field from Robinson et al. (2003), Penev et al. found that Zahn's linear scaling is more appropriate for modeling tidal dissipation. The right panel of Fig. 1 compares the different scalings with that obtained from the simulation. Zahn's scaling (dashed line) is closest to the simulation (solid line). This result is a consequence of the anisotropic nature of stellar convection (plumes) compared to classical Kolmogorov turbulence. 

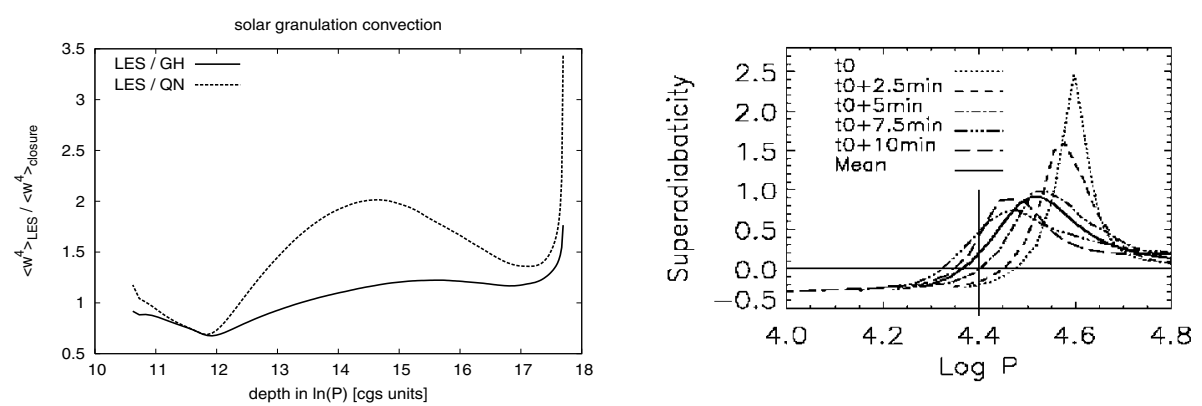

Figure 2. Left panel: the ratio of $\overline{w^{4}}$ to the QN and GH closure models (Kupka \& Robinson 2007a). Right panel: Instantaneous position of SAL over roughly half a granulation turnover time.

(d) Testing higher order turbulent closure models (Kupka \& Robinson 2007a). One of the main difficulties with Reynolds stress models of non-local stellar convection is when and how to close the hierarchical system of turbulence equations. The Gryanik \& Hartmann model (Gryanik \& Hartmann 2002) (denoted GH) has been applied to the planetary boundary layer and oceanic convection but has previously not been applied to realistic stellar convection. For sufficient integration time (at least 10 turnover times) the stellar simulations should provide a good representation of the higher order moments and can be used to compare higher order moments with appropriate closures written in terms of lower order moments. The left panel of Fig. 2 compares the ratio of the quasinormal approximation (QN) for $\overline{w^{4}}$ with its value directly computed from simulation and the same ratio using the GH closure $\overline{w^{4}}=3\left(1+(1 / 3)\left({\overline{w^{3}}}^{2} /{\overline{w^{2}}}^{3}\right)\right){\overline{w^{2}}}^{2}$ (see also Kupka \& Robinson 2007b). Away from the upper and lower boundaries the GH model differs by about $20 \%$ from unity while the QN approximation underestimates the fourth order moment by up to 200\% (see Kupka \& Robinson 2007a and Kupka \& Robinson 2007b for more details).

\section{Procyon A and the Sun}

\subsection{Basic facts}

Procyon A is one of the brightest objects in the sky. It is a 1.5 solar mass star that is about 10 light years away. Its outer convection zone occupies the outer $8 \%$ (by radius) and has a mass of $3 \mathrm{e}-5$ of the total mass of the star. By comparison, in the sun convection occurs in the outer $25 \%$ and the mass of the convection zone is about 0.02 of the solar mass. Our model of Procyon has an effective temperature of about $6500 \mathrm{~K}$, a surface gravity of about $90 \mathrm{~ms}^{-2}$ at $1.8 \mathrm{Gyr}$ and $\mathrm{X}$ and $\mathrm{Z}$ are equal to 0.97 and 0.018 . Note, because of very efficient helium diffusion during the stellar evolution, there is almost no helium in the simulation. It was incorrectly stated in Robinson et al. (2004) that there was $7 \%$ helium in the simulation model. In fact only 1.2\% by mass of helium was left. Although this will probably not make a significant change in the overall results, the effect of a higher helium abundance is presently being investigated, by suppressing helium diffusion in the initial model.

We have run Procyon simulations with computational boxes of widths $15 \mathrm{Mm}$ and $30 \mathrm{Mm}$. In each case there were only 60 grid points in the horizontal direction so that the larger box has poorer resolution. This did not seem to affect the overall properties of the simulations (velocities, pulsation of SAL etc). In both cases the depth is about $16 \mathrm{Mm}$ and there are 160 vertical grid points. 
Table 1. Characteristics of stellar simulations

\begin{tabular}{|c|c|c|c|c|}
\hline Model & $\mathrm{g}\left(\mathrm{ms}^{-2}\right)$ & $T_{\mathrm{eff}}(\mathrm{K})$ & $2 \times \mathrm{FWHM}(\mathrm{km})$ & $10 \mathrm{H}_{\mathrm{p}}(\mathrm{km})$ \\
\hline $\mathrm{K}-\mathrm{D}$ & 449 & 4609 & 600 & $550(530)$ \\
\hline $\mathrm{S}$ & 275 & 5800 & 1200 & $1400(1100)$ \\
\hline SG & 23 & 5000 & 13400 & $13.300(13,400)$ \\
\hline $\mathrm{P}$ & 89 & 6510 & 9000 & $6000(3750)$ \\
\hline Model & $w_{\max }{ }^{\prime \prime}\left(\mathrm{kms}^{-1}\right)$ & $\mathrm{T}_{\text {gran }}(\min )$ & $\mathrm{T}(\mathrm{SAL})(\mathrm{min})$ & $p_{\text {turb }} / p_{\text {gas }}$ \\
\hline K-D & 1.8 & 5.6 & $\mathrm{n} / \mathrm{a}$ & 6.5 \\
\hline $\mathrm{S}$ & 3 & 6.5 & $\mathrm{n} / \mathrm{a}$ & 12 \\
\hline SG & 3.3 & 68 & $50-70$ & $10-30$ \\
\hline $\mathrm{P}$ & 7 & 24 & $20-30$ & $20-60$ \\
\hline
\end{tabular}

\subsection{Flow patterns}

The most obvious differences between convection in the sun and Procyon A are that the large scale convective structures such as granules or plumes are much bigger in Procyon A (see www.astro.yale.edu/marjf for flow visulaizations). Even though the gravitational acceleration is about a third that of the sun, the granules appear to be about 9 times larger than those in the sun. Also the SAL appears to move in and out in a quasi-periodic manner. The right panel of Fig. 2 shows the SAL at a few instances over a cycle. By examining quantities (such as velocity, density or $\nabla_{\text {ad }}$ ) at different depths it seems that this oscillatory motion is confined to the region of granulation. If one looks at slightly deeper regions only noise can be seen. The SAL moves in and out over a distance of roughly half a pressure scale height in a time of between 20 and 30 minutes. We found similar behavior for the SAL in an 11.6 Gyr subgiant sun (SG) and in $\eta$-Bootis. However, no significant quasi-periodic motion was found for the sun (S) or a K-Dwarf (KD).

To illustrate how this pulsation is tied to the granulation we used the FWHM of the auto-correlation of the vertical velocity as the vertical length scale and the peak rms vertical velocity $w_{\max }{ }^{\prime \prime}$ as the velocity scale. Quantities listed in the Table 1 from left to right are the stellar model, twice the FWHM, 10 times the photospheric pressure scale height (the first value is from the simulation and the second from the initial stellar model), the maximum rms vertical velocity, the granule turnover time estimated as $T_{\text {gran }}=$ $2 \mathrm{FWHM} / w_{\max }^{\prime \prime}$, the approximate time for the SAL to move in and out once and finally the ratio of the peak turbulent pressure to the gas pressure. As the SAL moves in and out the peak turbulent pressure also varies quasi-periodically. The results imply that the pressure scale height in simulations tends to be about $30 \%$ larger than value in initial stellar models and that the period of SAL movement is similar to granule turnover time.

\section{Conclusions}

$3 \mathrm{D}$ radiative hydrodynamical simulations have become a viable tool for investigating the outer layers of sun-like stars with observed solar surface quantities being satisfactorily reproduced in the simulations. Interestingly some of the present stellar simulations show quasi- periodic oscillation with the amplitude strongest in the region dominated by granules (i.e. in the SAL) The origin of this oscillation is not clear but it may be due to the combined action of driving by a kappa-mechanism and granule overturning driven by radiative cooling. Further testing is needed to confirm that this is indeed a physical mechanism and not a numerical artifact such as a box eigenmode. We will run a series of addditional simulations with different depths and double the width of the box again to 
$60,000 \mathrm{~km}$. We will also look at the effect of the near absence of helium from the stellar model and the opacity dependence on temperature in the SAL.

\section{References}

Canuto, V.M. \& Mazzitelli, I., 1991, ApJ 370, 275

Chan, K.L. \& Sofia, S. 1989, ApJ 336, 1022

Goldreich, P. \& Keeley, D.A. 1989, ApJ 211, 934

Goodman, J. \& Oh, S.P. 1997, ApJ 486, 403

Gryanik, V.M. \& Hartmann, J. 2002, J. Atmos. Sci. 59, 2729

Guenther, D.B. \& Demarque, P. 1997, ApJ 484, 937

Kim, Y.-C. and Chan, K.L. 1998, ApJ 496, L121

Kupka, F. 2005, in Element Stratification in Stars: 40 Years of Atomic Diffusion, edts. G. Alecian, O. Richard and S. Vauclair, EAS Publications Series, Vol. 17, opp. 177

Kupka, F. \& Robinson, F.J. 2007a, MNRAS 374, 305

Kupka, F. \& Robinson, F.J. 2007b, this volume p. 74

Li, L.H., Robinson, F.J., Demarque, P., Sofia, S. and Guenther, D.B. 2002, ApJ 567, 1192

Penev, K., Sasselov, D., Robinson, F.J. \& Demarque, P. 2006, ApJ, in press

Robinson, F.J. \& Chan, K.L. 2004, Phys. of Fluids 15, 5, 1321

Robinson, F.J., Demarque, P., Li, L.H., Sofia, S., Kim, Y.-C., Chan, K.L. \& Guenther, D.B. 2003, MNRAS 340, 923

Robinson, F.J., Demarque, P., Li, L.H., Sofia, S., Kim, Y.-C., Chan, K.L. \& Guenther, D.B. 2004, MNRAS 347, 1208

Robinson, F.J. \& Sherwood, S.C. 2006, J. Atmos. Sci. 63, 1013

Stein, R.F. and Nordlund, A. 1998, ApJ 499, 914

Stein, R.F. and Nordlund, Å. 2000, Solar Phys. 192, 91

Straka, C.W., Demarque, P., Guenther, D.B., Li, L.H. \& Robinson, F.J. 2006, ApJ 636, 1078

Unno, W., \& Spiegel, E.A. 1966, PASJ 18, 85

Zahn, J.P., 1966, Ann. d'Ap 29, 489

\section{Discussion}

STEFFEn: Just for clarification: does your equation of state account for ionization?

Robinson: Yes - we use the opal opacities and equation of state which account for ionization.

BEDDING: We will observe Procyon in January 2007 in a multi-site asteroseismology campaign. Do you have a prediction for velocity observations integrated over the full disk, based on the oscillations found in your simulations?

RoBinson: By averaging horizontally in the region dominated by granulation (the SAL), it appears that the radial velocity varies periodically from $0.5 \mathrm{~km} / \mathrm{s}$ to $-0.5 \mathrm{~km} / \mathrm{s}$ over about 25 minutes. If this is assumed to be representative of the entire disk then we would expect to see a lateral shift of those spectral lines located at the depth of the SAL peak about their mean position.

LuDwoG: Rather a comment: Different from the findings of Robinson and collaborators our convection models of Procyon do not show pulsations other than p-modes of the computational box. Hence, we do not expect that observations of Procyon will show other oscillation signals than the expected solar-like oscillations.

Robinson: OK

Stein: Two comments: 1 . Turbulent viscosity will be highly anisotropic because the turbulent flow in such highly stratified stars is highly anisotropic. 2. A better treatment of radiation might improve the convection model.

Robinson: It might but by how much is not clear to me. A graduate student of Y.C. Kim at Yonsei University, is presently looking into this question. 